\title{
Quality of Chest Compressions Differs over Time between Advanced and Basic Life Support
}

\author{
Pär Lindblad1*, Annika Åström Victorén¹, Christer Axelsson², Bjarne Madsen Härdig3 \\ ${ }^{1}$ Department of Emergency, Värnamo County Hospital, Region Jönköpings län, Sweden \\ ${ }^{2}$ School of Health Sciences, University of Borås, Borås, Sweden \\ ${ }^{3}$ Physio-Control Sverige/Jolife AB, Ideon Science Park, Lund, Sweden \\ Email: *par.lindblad@rjl.se, annika.astrom.victoren@rjl.se,christer.axelsson@hb.se, \\ bjarne.madsen.hardig@physio-control.com
}

Received 6 November 2015; accepted 26 December 2015; published 29 December 2015

Copyright (C) 2015 by authors and Scientific Research Publishing Inc.

This work is licensed under the Creative Commons Attribution International License (CC BY). http://creativecommons.org/licenses/by/4.0/

(c) (i) Open Access

\section{Abstract}

Purpose: According to guideline recommendations, chest compressions (CC) during cardiopulmonary resuscitation (CPR) should be performed at a rate of 100 - 120 per minute, with a CC fraction (CCF) of $\geq \mathbf{8 0 \%}$. The aim of this work is to explore whether CC quality differs between advanced life support (ALS) and basic life support (BLS) performed by two rescuers. Method: Cardiopulmonary resuscitation was performed by two ambulance personnel in ten ALS and ten BLS manikin scenarios. Data from these scenarios were then compared with data on ten ALS cases from the clinical setting, all with non-shockable rhythms. Data from the first two 5-minute periods of CC were evaluated from impedance data (LIFEPAK 12 defibrillator monitors) using a modified Laerdal Skillmaster manikin. Quality parameters compared were: number of CC pauses (CCPs), total time of CC (\%), number of CC given and CC rate/min. Results: During the first 5 minutes, the BLS manikin scenarios had the highest number of CCPs, 15 (14 - 16), compared with the ALS manikin scenario, $14(13$ - 15), and the clinical ALS cases, 12 (10 - 15). The BLS scenario also had the highest CCFs, 81\% (77\% - 85\%), and number of CC, 450 (435 - 495), compared with the ALS manikin scenario, 75\% (64\% - 81\%) and 400 (365 - 444) respectively, and the clinical ALS cases, $63 \%$ $(50 \%-74 \%)$ and $408(306-489)$. The median rate of CC/min in the BLS scenario was 115 (110 120) compared with the ALS manikin scenario, 110 (106 - 115), and the clinical ALS cases, 130 (118 - 146). During the second 5-minute period, the BLS scenario had the highest number of CCPs, 16 (15 - 17), compared with 15 (14 - 16) for the ALS manikin scenario and 11 (11 - 12) for the clinical ALS cases. The CCF in the BLS setting was 79\% (75\% - 83\%), and the number of CC 455 (430 480), compared with the ALS manikin scenario, 79\% (74\% - 84\%) and 435 (395 - 480) respectively, and the clinical ALS cases, $71 \%(57 \%-77 \%)$ and $388(321-469)$ respectively. The median

\footnotetext{
${ }^{*}$ Corresponding author.
} 
CC rate was 118 (113 - 124) for BLS, 111 (105 - 120) for ALS manikins and 123 (103 - 128) CC/min for clinical ALS cases. Conclusion: None of the groups being studied could deliver CC at a rate of $100-120 \mathrm{CC} / \mathrm{min}$ or a CCF of $\geq 80 \%$ over the whole 10 -minute period in any of the resuscitation scenarios analyzed. However, BLS had the best compliance with CC quality recommendations according to the 2010 guidelines.

\title{
Keywords
}

\author{
Cardiac Arrest, External Chest Compression, Cardiac Resuscitation, Advanced Life Support, Basic \\ Life Support
}

\section{Introduction}

Cardio pulmonary resuscitation (CPR) is an important factor contributing to survival when treating a cardiac arrest caused by myocardial infarction [1]-[6]. Delivery of high-quality chest compressions (CC) is essential in this process and its effects on outcome have been studied in both experimental and out-of-hospital cardiac arrest (OHCA) studies [7]-[10]. The American Heart Association (AHA) 2010 resuscitation guidelines [11] focused on high-quality CC and emphasized that rescuers should perform CC at an adequate rate (100 - $120 \mathrm{CC} / \mathrm{min})$, adequate depth $(5-6 \mathrm{~cm})$, and with a minimum of interruptions, while avoiding leaning and hyperventilation. After 30 CC, they should introduce a 3-second pause to allow for two ventilations. Furthermore, if there are multiple rescuers, they should rotate every 2 minutes to avoid fatigue [3]. In July 2013, the AHA published a consensus statement that, besides previously described parameters, emphasized that CC fraction (CCF; \% of time) should be at least $80 \%$ [12]. Chest compression fraction is the proportion of time that CC is performed during a cardiac arrest.

Previous studies have shown difficulties in maintaining CC quality, if performed manually, in both training and clinical settings [13]-[15]. The treatment recommendations for adult OHCA can be taught as basic life support (BLS) or advanced life support (ALS) [6] [16]-[19]. Basic life support includes maintenance of airway patency, breathing support and CC, and may also include using an automatic external defibrillator. When performing ALS, in addition to the tasks done during BLS, the use of manual defibrillators, management of intravenous drugs and advanced airway managements are recommended [17] [20]. The purpose of performing ALS using these more advanced treatments is to improve cardiac arrest outcomes [11]; however, if there are few persons involved in a resuscitation case, this may affect CC quality as there are more tasks to be done. Furthermore, ambulance personnel have to perform CPR in many different situations and this has been shown to influence both the quality and continuity of the care given [8] [21] [22]. Previous studies comparing ALS with BLS in the pre-hospital setting have failed to show any clinical outcome benefits [23] [24].

The aim of the present study is to explore whether there are quality differences between CC, performed by two ambulance personnel, in two 10 minutes manikin scenarios of ALS, and BLS compared to ALS in the OHCA setting [11].

\section{Material and Methods}

\subsection{Study Design}

\subsubsection{Study Population and Training}

Life support, in ten ALS and ten BLS manikin scenarios, was performed by two ambulance personnel. Quality parameters from these scenarios were then compared with parameters from ten clinical ALS cases. Participants for the BLS and ALS manikin scenarios were recruited in a randomized manner among the ambulance staff (ambulance personnel from the Department of Emergency, Medical Service (EMS), Värnamo County Hospital, Värnamo, Sweden) by the first author (P.L.). The ambulance personnel volunteered freely and did not receive any financial compensation. All participants had rehearsed ALS and BLS skills within the 3 months prior to the study. They were allowed to test the manikin to make sure that they could reach the correct depth. Before the manikin scenarios each pair were able to arrange how ALS and BLS tasks should be divided between 
them.

\subsubsection{Manikin Setting}

All manikin scenarios were performed on a Skillmaster 4000 manikin (Laerdal, Stavanger, Norway). The BLS scenario started with immediate CC and ventilation using bag valve mask ventilation, followed by a rhythm check every 2 minutes as recommended [3] [6]. All ALS scenarios started with immediate CC and bag valve mask ventilation, after the airway was secured, using a laryngeal mask (I-gel 4; Intersurgical Ltd, Wokingham, UK) with a stethoscope to confirm the correct placement of the mask. A peripheral venous line was established, secured and its placement confirmed by injecting saline. Sham injection of epinephrine for administration every 4 minutes was drawn up in syringes.

In all scenarios participants used the defibrillator in manual mode and in all scenarios the rhythm was nonshockable. The manikin scenarios took place indoors on the floor of the ambulance station.

\subsubsection{Clinical Setting}

During the period between October 2010 and May 2012 a total of 175 cardiac arrest cases were registered in Jönköping County, Sweden [25]. Of these, CODE-STAT 8.0 (Physio-Control, Redmond, WA, USA) data were available for 55 cases. Ten patients had non-shockable rhythm during the first 10 minutes of the resuscitation. These were selected to match the manikin scenarios described above. All cases were treated according to the 2010 European Resuscitation Council (ERC) guidelines [19] for ALS, with the defibrillator in manual mode.

\subsubsection{Equipment and Analysis of Data}

For the manikin scenario, an ALS Skillmaster 4000 was used, with two cords placed at a depth of $4.5 \mathrm{~cm}$ inside the manikin. This modification enabled impedance recordings each time a CC exceeded the $4.5 \mathrm{~cm}$ depth. The two cords were connected to the electrodes on the manikin and then to the defibrillator pad cable which registered the impedance wave forms [26] [27] using LIFEPAK 12 defibrillators (Physio-Control, Redmond, WA, USA).

Chest compression quality parameters, using CODE-STAT 8.0, from two 5-minute intervals of the BLS, as well as the ALS manikin scenarios and the ten clinical ALS cases were evaluated.

Chest compression pauses (CCPs) $>2.5$ seconds (n), CCF, number of CC (n), and CC rate (compressions/ minute) were analysed. Measurements of time and time intervals were done using Cardio Calipers software (Iconico, New York, NY, USA).

\subsection{Statistics}

All data are reported as median values and inter-quartile ranges (IQRs) but figure also show error bars indicating min and max values. For statistical comparison, Mann-Whitney U-test was used and a $p$-value $<0.05$ considered significant.

\section{Results}

None of ambulance personnel pairs could produce both a CC rate between 100 - 120 and a CCF $\geq 80 \%$ over the whole 10-minute period in any scenario, no matter which algorithm was followed.

\subsection{Chest Compression Pauses}

During the first 5 minutes the lowest number of CCPs; 12 (10 - 15), was noted in the ALS clinical setting compared with $14(13-15)$ in the ALS manikin scenario, and $15(14-16)$ for the BLS scenario. This difference in CCPs increased during the period of 6 - 10 minutes. For this period, the number was $11(11-12)$ in the ALS clinical setting; 15 (14 - 16) in the ALS manikin scenario and 16 (15 - 17) in the BLS scenario (Figure 1(a) and Figure 1(b); Table 1).

\subsection{Chest Compression Fraction}

During the first 5 minutes, the highest CCF, of 81\% (77\% - 85\%), was seen in the BLS scenario, compared with $75 \%(64 \%-81 \%)$ in the ALS manikin scenario and 63\% (50\% - 74\%) in the ALS clinical setting. This difference 


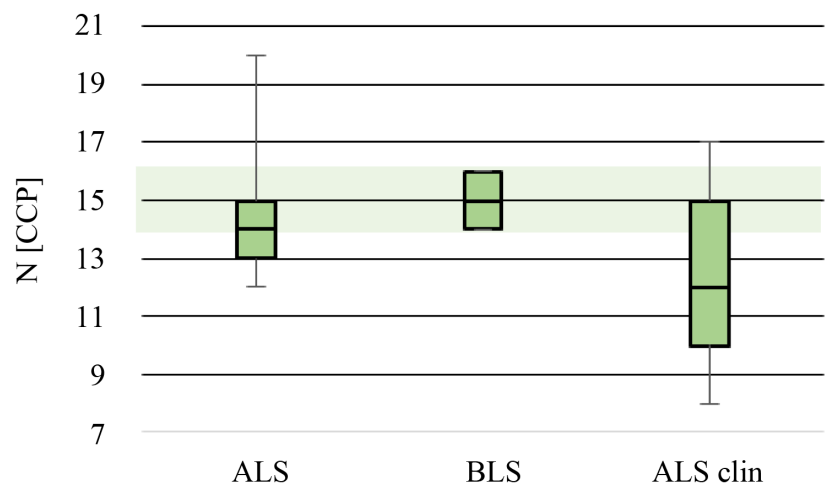

(a)

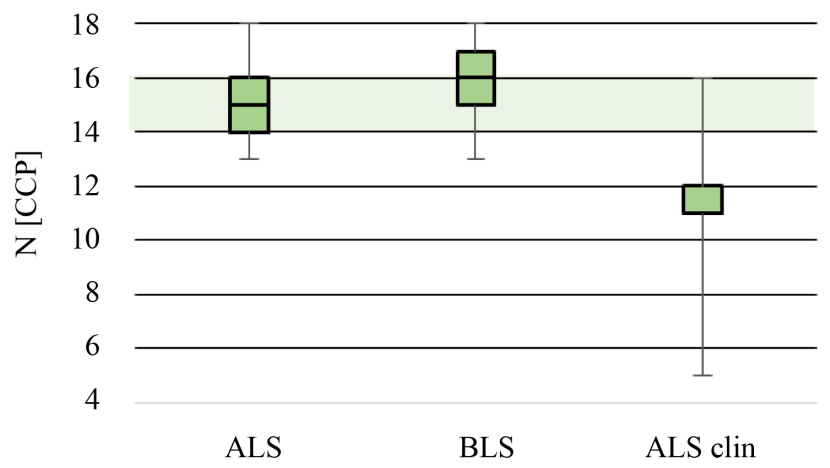

(b)

Figure 1. (a): Number of chest compression (CC) pauses (CCP) during the first 5 minutes of cardiopulmonary resuscitation (CPR) for the manikin scenarios and the clinical cases. The light green area in the Figure shows American Heart Association (AHA) and European Resuscitation Council (ERC) guideline values for 2010, with a recommended CC rate of 100 - 120 $\mathrm{CC} / \mathrm{min}$. (b): Number of CCPs during the second 5-minute period for the manikin scenarios and the clinical cases. The light green area shows AHA and ERC guidelines 2010, with a recommended CC rate of $100-120 \mathrm{CC} / \mathrm{min}$. Data is presented as median \pm 1 st-3rd inter-quartile ranges (IQRs) and the error bars indicate min and max values. ALS = advanced life support performed in a manikin setting; BLS = basic life support performed in the manikin setting; ALS clin = advanced life support performed in the clinical setting.

Table 1. Chest compression (CC) parameters during the first and the second 5-minute period of cardiopulmonary resuscitation (CPR). Data are given as medians and inter-quartile ranges (IQRs). Statistical comparison was made using unpaired Mann-Whitney U-test. A $p$-value $<0.05$ was considered significant.

\begin{tabular}{|c|c|c|c|c|c|c|}
\hline CPR 1-5 minutes & ALS & BLS & $P$-value & ALS clinical & $\begin{array}{c}P \text {-value } \\
\text { (ALS) }\end{array}$ & $P$-value (BLS) \\
\hline Chest compressions pause (n) & $14(13-15)$ & $15(14-16)$ & 0.567 & $12(10-15)$ & 0.015 & 0.0002 \\
\hline Chest compressions fraction (\%) & $75 \%(64 \%-81 \%)$ & $81 \%(77 \%-85 \%)$ & 0.003 & $63 \%(50 \%-74 \%)$ & $<0.0001$ & $<0.0001$ \\
\hline Chest compressions (n) & $400(365-444)$ & $450(435-495)$ & $<0.0001$ & $408(306-489)$ & 0.937 & 0.015 \\
\hline Chest compressions (beats/min) & $110(106-115)$ & $115(110-120)$ & 0.029 & $130(118$ - 146) & $<0.0001$ & $<0.0001$ \\
\hline CPR 6-10 minutes & ALS & BLS & $P$-value & ALS clinical & $\begin{array}{c}P \text {-value } \\
\text { (ALS) }\end{array}$ & $P$-value (BLS) \\
\hline Chest compressions pause (n) & $15(14-16)$ & $16(15-17)$ & 0.445 & $11(11-12)$ & $<0.0001$ & $<0.0001$ \\
\hline Chest compressions fraction (\%) & $79 \%(74 \%-84 \%)$ & $79 \%(75 \%-83 \%)$ & 0.97 & $71 \%(57 \%-77 \%)$ & 0.001 & $<0.0001$ \\
\hline Chest compressions (n) & $435(395-480)$ & $455(430-480)$ & 0.136 & $388(321$ - 469) & 0.075 & 0.003 \\
\hline Chest compressions (beats/min) & $111(105-120)$ & $118(113-124)$ & 0.022 & $123(103-128)$ & 0.038 & 0.298 \\
\hline
\end{tabular}


persisted throughout the procedure. When comparing both manikin scenarios there was a significant difference also at 6 - 10 minutes: clinical ALS cases; 71\% (55\% - 77\%) compared with the ALS manikin scenario; 79\% (74\% - 84\%) and BLS scenario; 79\% (75\% - 83\%) (Figure 2(a) and Figure 2(b); Table 1).

\subsection{Number of Chest Compressions Given}

During the first 1 - 5 minutes the highest number of CC was given in the BLS manikin scenarios ( $\mathrm{n}=450$ (435 495). There was no difference between the two ALS scenarios. In the ALS clinical setting, 408 (306 - 489) and in the ALS manikin scenario 400 (365 - 444) compressions were given. This difference persisted when comparing the different scenarios at 6 - 10 minutes: BLS manikin scenario; $n=455(430-480)$, versus ALS manikin scenario; $n=435$ (395 - 480) and the ALS clinical setting; $n=388$ (321 - 469) (Figure 3(a) and Figure 3(b), Table 1).

\subsection{Chest Compression Rate}

During the first 5 minutes the highest CC rate (130 (118 - 146) was given in the ALS clinical setting compared with $115(110$ - 120) for the BLS manikin scenario and 110 (106 - 115) for the ALS manikin scenario. For both manikin scenarios, this was within the 2010 guideline [28] range. The difference in rate persisted when comparing the different scenarios during 6 - 10 minutes of CC: ALS clinical setting; 123 (103 - 128) versus BLS manikin scenario; 118 (113 - 124) and ALS manikin scenario; 111 (105 - 120) (Figure 4(a) and Figure 4(b), Table 1).

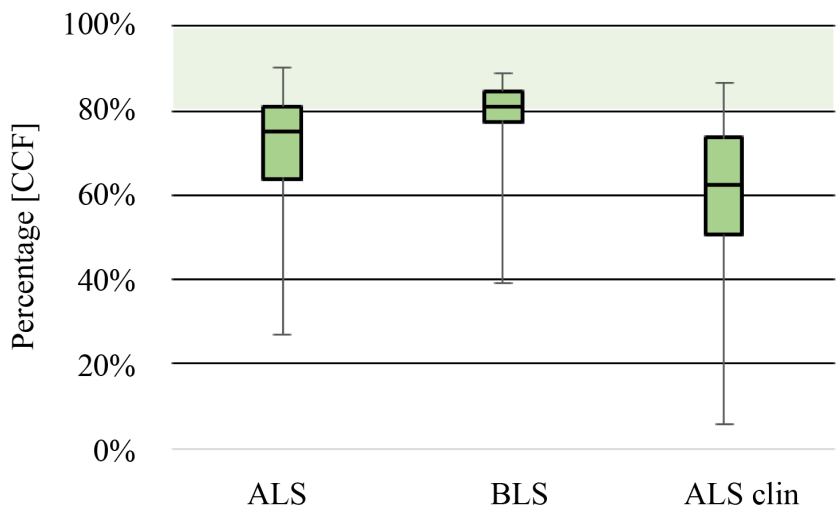

(a)

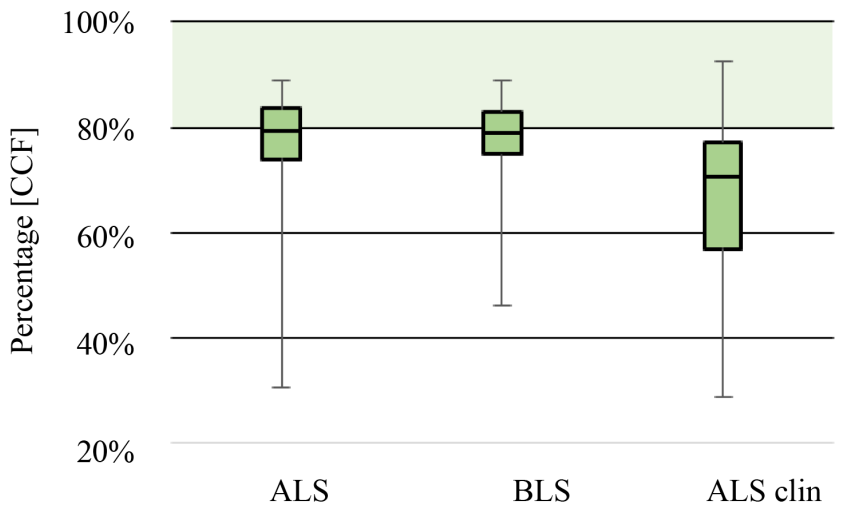

(b)

Figure 2. (a): The chest compression (CC) fraction (CCF) during the first 5 minutes of cardiopulmonary resuscitation (CPR) for the manikin scenarios and the clinical cases. (b): The CCF during the second 5-minute period. The light green area in the Figure shows a CCF of $\geq 80 \%$ according to the American Heart Association (AHA) 2013 update. Data is presented as median \pm 1 st-3rd inter-quartile ranges (IQRs) and the error bars indicate min and max values. ALS = advanced life support using a manikin setting; BLS = basic life support performed in a manikin setting; ALS clin = advanced life support performed in the clinical setting. 

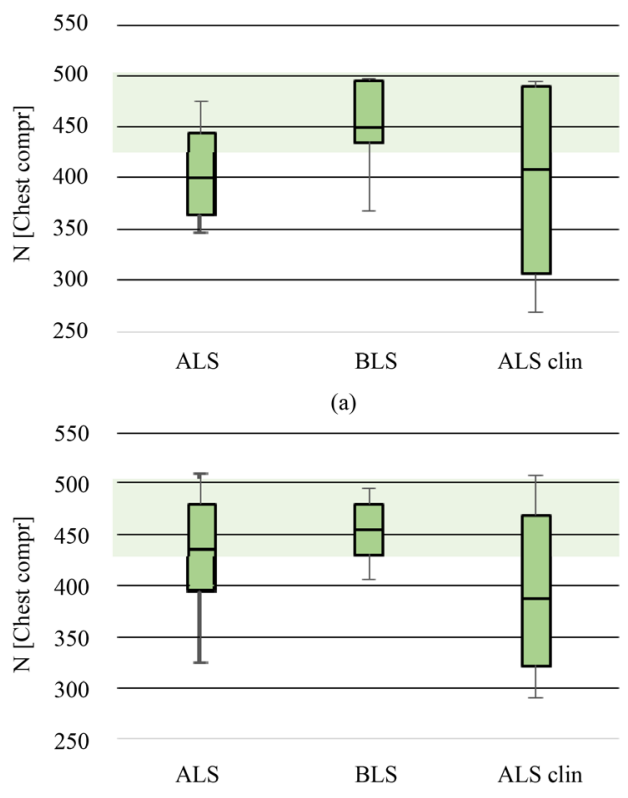

(b)

Figure 3. (a): The number of chest compressions (CC) during the first 5 minutes of cardiopulmonary resuscitation (CPR) for the manikin scenarios and the clinical cases. (b): The number of CC during the second 5-minute period. The light green area in the Figure shows American Heart Association (AHA) and European Resuscitation Council (ERC) guideline rates for 2010, with a recommended CC rate of $100-120 \mathrm{CC} / \mathrm{min}$. Data is presented as median \pm 1 st-3rd inter-quartile ranges (IQRs) and the error bars indicate min and max values. ALS = advanced life support performed in a manikin setting; BLS = basic life support performed in a manikin setting; ALS clin = advanced life support performed in the clinical cases.

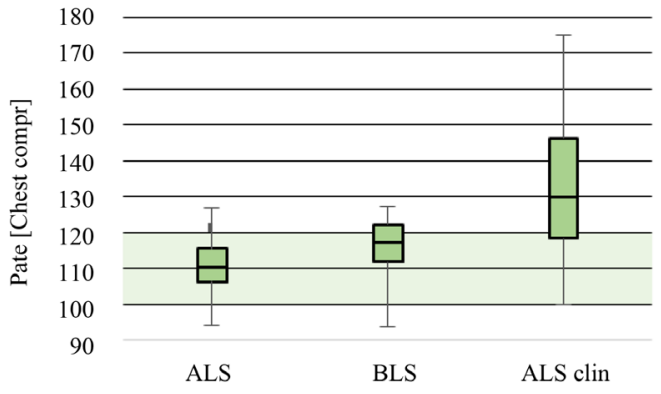

(a)

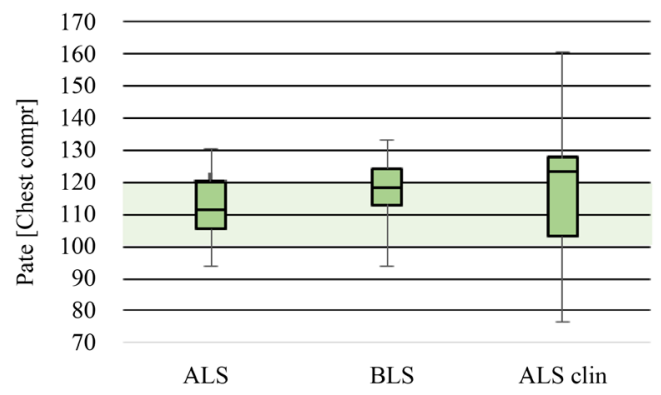

(b)

Figure 4. (a): The chest compression (CC) rate during the first 5 minutes of cardiopulmonary resuscitation (CPR) for the manikin scenarios and clinical cases. (b): The CC rate during the second 5-minute period. The light green area in the Figure shows American Heart Association (AHA) and European Resuscitation Council (ERC) guideline rates for 2010. Data is presented as median \pm 1 st-3rd inter-quartile ranges (IQRs) and the error bars indicate min and max values. ALS = advanced life support performed in a manikin setting; BLS = basic life support performed in a manikin setting; ALS clin = advanced life support performed in the clinical cases. 


\section{Discussion}

The results from this study show that none of the included ambulance personnel in any of the three scenarios explored succeeded in maintaining a CC rate of 100 - 120 and a CCF of $\geq 80 \%$ over the whole 10 -minute period. This study also shows that there is a gap between current knowledge of CPR and the quality expected to be performed in clinical practice [12], even though high-quality CPR is one of the primary components in resuscitation. This may offer one explanation for the significant variability in survival rates both across and within pre-hospital and in-hospital settings [5] [29].

Chest compression pauses for ventilation are essential and accepted when using the 30:2 algorithm. However, it is important to prevent other, potentially avoidable pauses since these have been shown to be associated with lower return of spontaneous circulation (ROSC) and survival rate [30]. The BLS manikin scenario had more CCPs compared with the ALS manikin scenario and ALS clinical setting. The reason for this was that BLS followed 2010 guidelines substantially better than did either the ALS manikin or the clinical setting. Previous study has linked a low CCF to frequent CCPs [31]. However, in this study the CCP length, rather than the CCP frequency, had a negative effect on CCF, particularly in the ALS clinical setting. Our study shows that even with the clarification of the manikin setting, there were significant differences in CCPs during the first 5 minutes of CRP when we compared the ALS and BLS scenarios.

For the first 5 minutes in the BLS manikin scenario, the CCF was $>80 \%$ and significantly greater compared with the ALS manikin and the ALS clinical scenarios. The difference in CCF between BLS and ALS may be explained by the ALS algorithm, as well as the greater number of activities that need to be done during ALS, which might be difficult to perform when only two personnel are available [20]. During the second 5-minute period, once the airway was secured and a peripheral venous line was established the CCF improved in both the ALS manikin scenario and the ALS clinical setting. Previous studies have also shown that CCF is often poor in a pre-hospital setting, as seen in our study [32], and CCF is important for outcome [7], which may explain why ALS failed to improve outcome compared with BLS [22]-[24]. To maintain CPR quality in ALS, which involves more handling, it is important to evaluate ALS training, particularly as OHCA is rare for ambulance personnel [33].

In this study, when the personnel performed BLS they achieved the highest number of CC, both during the first and during the second 5-minute period. They achieved the guideline-recommended number of CC of 100 120/min, despite a higher number of pauses. Among the ALS cases, a higher CC rate was achieved for the ALS clinical compared with the ALS manikin scenario. Previous studies [34] have described the negative effect of a suboptimal CC rate of $<100 \mathrm{CC} / \mathrm{min}$ and a CC rate of $>120 \mathrm{CC} / \mathrm{min}$, which might lead to unfavorable coronary perfusion due to shorter decompression time [35] and thus reduce the possibility of successful defibrillation [36]. We noted that the median rate for CC for ALS and BLS scenarios followed the 2010 guidelines [28] and that the CC rate in the ALS clinical setting decreased over time. The greater number of activities and handling in the ALS scenario did not have any impact on CC rate in the manikin setting, which is in agreement with an earlier study [37]. So the high CC rate seen in the ALS clinical setting could be a result of lack of leadership skills and team factors [38] [39]. Also, the stressful situation of a cardiac arrest could affect the ambulance personnel [40] although the impact of acute stress on performance during CPR is still debated [41].

This study shows an easy way to review CPR quality parameters by counting the number of CC over 5 minutes. When the median for number of CC is between 430 and 500 CC during 5 minutes we found that also CCP, CCF and CC rate are within the accepted range. However, more studies need to verify whether number of CC on its own is a fast and uncomplicated way to evaluate the quality of CCP, and CCF and CC rate (Figures 1-4).

\section{Limitations}

There are some limitations to our study. Data on the clinical ALS and OHCA cases were collected retrospectively, and more cases could not be used because of lacking data and missing trans-thoracic impedance data, which may have influenced the results. This study was performed in one single center and the manikin scenarios were simulated by two ambulance personnel starting the resuscitation. Cardiac arrest in the clinical environment can include support from more personnel and in that case the results might be different. A different organization and number of ambulance personnel may therefore render different results. The use of a metronome would be one way to control the compression rate but there was none used in this study. 


\section{Conclusion}

None of the groups being studied could achieve a CC rate of $100-120$ CC/min and a CCF of $\geq 80 \%$ over the entire 10-minute period in each resuscitation scenario analyzed. In this respect, BLS had the best compliance with the 2010 guidelines.

\section{Acknowledgements}

Advanced life support (ALS), American Heart Association (AHA), Basic life support (BLS), Chest compression fractions (CCF), Chest compressions (CC), Chest compression pauses (CCP), Out-of-hospital cardiac arrest (OHCA), Cardiopulmonary resuscitation (CPR), Medical Service (EMS), Inter-quartile ranges (IQRs).

\section{References}

[1] Vukmir, R.B. (2006) Survival from Prehospital Cardiac Arrest Is Critically Dependent upon Response Time. Resuscitation, 69, 229-234. http://dx.doi.org/10.1016/j.resuscitation.2005.08.014

[2] Sasson, C., Rogers, M.A., Dahl, J. and Kellermann, A.L. (2010) Predictors of Survival from Out-of-Hospital Cardiac Arrest: A Systematic Review and Meta-Analysis. Circulation: Cardiovascular Quality and Outcomes, 3, 63-81. http://dx.doi.org/10.1161/CIRCOUTCOMES.109.889576

[3] Travers, A.H., Rea, T.D., Bobrow, B.J., Edelson, D.P., Berg, R.A., et al. (2010) Part 4: CPR Overview: 2010 American Heart Association Guidelines for Cardiopulmonary Resuscitation and Emergency Cardiovascular Care. Circulation, 122, S676-S684. http://dx.doi.org/10.1161/CIRCULATIONAHA.110.970913

[4] Bobrow, B.J., Clark, L.L., Ewy, G.A., Chikani, V., Sanders, A.B., et al. (2008) Minimally Interrupted Cardiac Resuscitation by Emergency Medical Services for Out-of-Hospital Cardiac Arrest. JAMA, 299, 1158-1165. http://dx.doi.org/10.1001/jama.299.10.1158

[5] Berdowski, J., Berg, R.A., Tijssen, J.G.P. and Koster, R.W. (2010) Global Incidences of Out-of-Hospital Cardiac Arrest and Survival Rates: Systematic Review of 67 Prospective Studies. Resuscitation, 81, 1479-1487. http://dx.doi.org/10.1016/j.resuscitation.2010.08.006

[6] Nolan, J.P., Soar, J., Zideman, D.A., Biarent, D. and Bossaert, L.L. (2010) European Resuscitation Council Guidelines for Resuscitation 2010 Section 1. Executive Summary. Resuscitation, 81, 1219-1276. http://dx.doi.org/10.1016/j.resuscitation.2010.08.021

[7] Vaillancourt, C., Everson-Stewart, S., Christenson, J., Andrusiek, D., Powell, J., et al. (2011) The Impact of Increased Chest Compression Fraction on Return of Spontaneous Circulation for Out-of-Hospital Cardiac Arrest Patients Not in Ventricular Fibrillation. Resuscitation, 82, 1501-1507. http://dx.doi.org/10.1016/j.resuscitation.2011.07.011

[8] Olasveengen, T.M., Wik, L., Kramer-Johansen, J., Sunde, K., Pytte, M., et al. (2007) Is CPR Quality Improving? A Retrospective Study of Out-of-Hospital Cardiac Arrest. Resuscitation, 75, 260-266. http://dx.doi.org/10.1016/j.resuscitation.2007.04.016

[9] Ewy, G.A., Zuercher, M., Hilwig, R.W., Sanders, A.B., Berg, R.A., et al. (2007) Improved Neurological Outcome with Continuous Chest Compressions Compared with 30:2 Compressions-to-Ventilations Cardiopulmonary Resuscitation in a Realistic Swine Model of Out-of-Hospital Cardiac Arrest. Circulation, 116, 2525-2530. http://dx.doi.org/10.1161/CIRCULATIONAHA.107.711820

[10] Field, R.A., Soar, J., Davies, R.P., Akhtar, N. and Perkins, G.D. (2012) The Impact of Chest Compression Rates on Quality of Chest Compressions-A Manikin Study. Resuscitation, 83, 360-364. http://dx.doi.org/10.1016/j.resuscitation.2011.07.012

[11] Bhanji, F., et al. (2010) Part 16: Education, Implementation, and Teams: 2010 American Heart Association Guidelines for Cardiopulmonary Resuscitation and Emergency Cardiovascular Care. Circulation, 122, S920-S933. http://dx.doi.org/10.1161/CIRCULATIONAHA.110.971135

[12] Meaney, P.A., et al. (2013) Cardiopulmonary Resuscitation Quality: Improving Cardiac Resuscitation Outcomes Both Inside and Outside the Hospital: A Consensus Statement from the American Heart Association. Circulation, 128, 417-435. http://dx.doi.org/10.1161/CIR.0b013e31829d8654

[13] Foo, N.-P., Chang, J.-H., Lin, H.-J. and Guo, H.-R. (2010) Rescuer Fatigue and Cardiopulmonary Resuscitation Positions: A Randomized Controlled Crossover Trial. Resuscitation, 81, 579-584. http://dx.doi.org/10.1016/j.resuscitation.2010.02.006

[14] Fried, D.A., et al. (2011) The Prevalence of Chest Compression Leaning during In-Hospital Cardiopulmonary Resuscitation. Resuscitation, 82, 1019-1024. http://dx.doi.org/10.1016/j.resuscitation.2011.02.032

[15] Haque, I.U., Udassi, J.P., Udassi, S., Theriaque, D.W., Shuster, J.J. and Zaritsky, A.L. (2008) Chest Compression 
Quality and Rescuer Fatigue with Increased Compression to Ventilation Ratio during Single Rescuer Pediatric CPR. Resuscitation, 79, 82-89. http://dx.doi.org/10.1016/j.resuscitation.2008.04.026

[16] Berg, R.A., et al. (2010) Part 5: Adult Basic Life Support: 2010 American Heart Association Guidelines for Cardiopulmonary Resuscitation and Emergency Cardiovascular Care. Circulation, 122, S685-S705. http://dx.doi.org/10.1161/CIRCULATIONAHA.110.970939

[17] Neumar, R.W., et al. (2010) Part 8: Adult Advanced Cardiovascular Life Support: 2010 American Heart Association Guidelines for Cardiopulmonary Resuscitation and Emergency Cardiovascular Care. Circulation, 122, S729-S767. http://dx.doi.org/10.1161/CIRCULATIONAHA.110.970988

[18] Koster, R.W., et al. (2010) European Resuscitation Council Guidelines for Resuscitation 2010 Section 2. Adult Basic Life Support and Use of Automated External Defibrillators. Resuscitation, 81, 1277-1292. http://dx.doi.org/10.1016/j.resuscitation.2010.08.009

[19] Deakin, C.D., et al. (2010) European Resuscitation Council Guidelines for Resuscitation 2010 Section 4. Adult Advanced Life Support. Resuscitation, 81, 1305-1352. http://dx.doi.org/10.1016/j.resuscitation.2010.08.017

[20] Deakin, C.D., et al. (2010) Part 8: Advanced Life Support: 2010 International Consensus on Cardiopulmonary Resuscitation and Emergency Cardiovascular Care Science with Treatment Recommendations. Resuscitation, 81, e93-e174. http://dx.doi.org/10.1016/j.resuscitation.2010.08.027

[21] Kampmeier, T.G., et al. (2014) Chest Compression Depth after Change in CPR Guidelines-Improved but Not Sufficient. Resuscitation, 85, 503-508. http://dx.doi.org/10.1016/j.resuscitation.2013.12.030

[22] Olasveengen, T.M., Wik, L. and Steen, P.A. (2008) Quality of Cardiopulmonary Resuscitation before and during Transport in Out-of-Hospital Cardiac Arrest. Resuscitation, 76, 185-190. http://dx.doi.org/10.1016/j.resuscitation.2007.07.001

[23] Bakalos, G., et al. (2011) Advanced Life Support versus Basic Life Support in the Pre-Hospital Setting: A Meta-Analysis. Resuscitation, 82, 1130-1137. http://dx.doi.org/10.1016/j.resuscitation.2011.04.006

[24] Sanghavi, P., Jena, A.B., Newhouse, J.P. and Zaslavsky, A.M. (2015) Outcomes after Out-of-Hospital Cardiac Arrest Treated by Basic vs Advanced Life Support. JAMA Internal Medicine, 175, 196-204. http://dx.doi.org/10.1001/jamainternmed.2014.5420

[25] Wang, H.E., Simeone, S.J., Weaver, M.D. and Callaway, C.W. (2009) Interruptions in Cardiopulmonary Resuscitation from Paramedic Endotracheal Intubation. Annals of Emergency Medicine, 54, 645-652. http://dx.doi.org/10.1016/j.annemergmed.2009.05.024

[26] Stecher, F.S., Olsen, J.A., Stickney, R.E. and Wik, L. (2008) Transthoracic Impedance Used to Evaluate Performance of Cardiopulmonary Resuscitation during Out of Hospital Cardiac Arrest. Resuscitation, 79, 432-437. http://dx.doi.org/10.1016/j.resuscitation.2008.08.007

[27] Alonso, E., et al. (2015) Reliability and Accuracy of the Thoracic Impedance Signal for Measuring Cardiopulmonary Resuscitation Quality Metrics. Resuscitation, 88, 28-34.

[28] Lim, S.H., et al. (2010) Part 7: CPR Techniques and Devices: 2010 International Consensus on Cardiopulmonary Resuscitation and Emergency Cardiovascular Care Science with Treatment Recommendations. Resuscitation, 81, e86-92. http://dx.doi.org/10.1016/j.resuscitation.2010.08.026

[29] Nichol, G., et al. (2008) Regional Variation in Out-of-Hospital Cardiac Arrest Incidence and Outcome FREE. JAMA, 300, 1423-1431. http://dx.doi.org/10.1001/jama.300.12.1423

[30] Brouwer, T., Walker, R., Koster, R. and Chapman, F. (2013) Association between Potentially Avoidable Pauses in Chest Compressions and Survival from Out-of-Hospital Cardiac Arrest. Resuscitation, 84, S3. http://dx.doi.org/10.1016/j.resuscitation.2013.08.024

[31] Aufderheide, T.P. and Lurie, K.G. (2004) Death by Hyperventilation: A Common and Life-Threatening Problem during Cardiopulmonary Resuscitation. Critical Care Medicine, 32, S345-S351. http://dx.doi.org/10.1097/01.CCM.0000134335.46859.09

[32] Wik, L., et al. (2005) Quality of Cardiopulmonary Resuscitation during Out-of-Hospital Cardiac Arrest. JAMA, 293, 299-304. http://dx.doi.org/10.1001/jama.293.3.299

[33] Dyson, K., Bray, J., Smith, K., Bernard, S., Straney, L. and Finn, J. (2015) Paramedic Exposure to Out-of-Hospital Cardiac Arrest Is Rare and Declining in Victoria, Australia. Resuscitation, 89, 93-98. http://dx.doi.org/10.1016/j.resuscitation.2015.01.023

[34] Abella, B.S., et al. (2005) Chest Compression Rates during Cardiopulmonary Resuscitation Are Suboptimal: A Prospective Study during in-Hospital Cardiac Arrest. Circulation, 111, 428-434. http://dx.doi.org/10.1161/01.CIR.0000153811.84257.59 
[35] Wolfe, J.A., et al. (1988) Physiologic Determinants of Coronary Blood Flow during External Cardiac Massage. The Journal of Thoracic and Cardiovascular Surgery, 95, 523-532.

[36] Reynolds, J.C., Salcido, D.D. and Menegazzi, J.J. (2010) Coronary Perfusion Pressure and Return of Spontaneous Circulation after Prolonged Cardiac Arrest. Prehospital Emergency Care, 14, 78-84. http://dx.doi.org/10.3109/10903120903349796

[37] Wik, L., et al. (2014) Manual vs. Integrated Automatic Load-Distributing Band CPR with Equal Survival after out of Hospital Cardiac Arrest. The Randomized CIRC Trial. Resuscitation, 85, 741-748. http://dx.doi.org/10.1016/j.resuscitation.2014.03.005

[38] Tschan, F., Vetterli, M., Semmer, N.K., Hunziker, S. and Marsch, S.C.U. (2011) Activities during Interruptions in Cardiopulmonary Resuscitation: A Simulator Study. Resuscitation, 82, 1419-1423. http://dx.doi.org/10.1016/j.resuscitation.2011.06.023

[39] Donoghue, A.J., Durbin, D.R., Nadel, F.M., Stryjewski, G.R., Kost, S.I. and Nadkarni, V.M. (2009) Effect of High-Fidelity Simulation on Pediatric Advanced Life Support Training in Pediatric House Staff: A Randomized Trial. Pediatric Emergency Care, 25, 139-144. http://dx.doi.org/10.1097/PEC.0b013e31819a7f90

[40] Krarup, N.H., et al. (2011) Quality of Cardiopulmonary Resuscitation in Out-of-Hospital Cardiac Arrest Is Hampered by Interruptions in Chest Compressions-A Nationwide Prospective Feasibility Study. Resuscitation, 82, 263-269. http://dx.doi.org/10.1016/j.resuscitation.2010.11.003

[41] Hunziker, S., Semmer, N.K., Tschan, F., Schuetz, P., Mueller, B. and Marsch, S. (2012) Dynamics and Association of Different Acute Stress Markers with Performance during a Simulated Resuscitation. Resuscitation, 83, 572-578. http://dx.doi.org/10.1016/j.resuscitation.2011.11.013 